\title{
UNASUR en el escenario del multilateralismo latinoamericano: luces y sombras
}

Anabella Busso*

\section{RESUMEN}

Latinoamérica, y Sudamérica en particular, afrontaron un escenario diferente en la primera década del siglo xxi. Entre los cambios producidos se destaca la aparición del denominado "regionalismo poshegemónico". En ese marco surgieron nuevos escenarios de cooperación entre los Estados de la región. Esta situación reabrió viejos debates, e instauró nuevos, en torno a cuestiones tales como: si estos procesos presentaban nuevas instancias de integración; si asistíamos a un nuevo multilateralismo latinoamericano; si dicho multilateralismo tenía características distintivas y qué impacto tenían estos procesos en relación con los vínculos con las potencias regionales y extrarregionales. En este artículo pretendemos avanzar algunas reflexiones sobre el caso específico de la Unión de Naciones del Sur (UnASUR), tratando de dar respuesta a los interrogantes antes mencionados. A partir del presente análisis, es posible señalar que se introdujo una práctica singular que ha dado lugar al multilateralismo sudamericano. El mismo se caracteriza, entre otras particularidades, por constituir un proceso de concertación política o, en su defecto, uno de integración multifuncional, distanciándose de perspectivas tradicionales, propias de las visiones economicistas de los procesos de integración. No obstante, también se debe mencionar que dicho proceso no fue lineal ni ha estado exento de dificultades. Así, UNASUR ha transitado un quinquenio de luces $\mathrm{y}$ un quinquenio de sombras, manifestando,

\footnotetext{
* Máster en Ciencias Sociales, Facultad Latinoamericana de Ciencias Sociales -FLACso- (Argentina). Investigadora de Conicet. Profesora Titular de Política Internacional y Política Internacional Latinoamericana, Facultad de Ciencia Política y Relaciones Internacionales, Universidad Nacional de Rosario, Rosario (Argentina). anabella.busso@fcpolit.unr.edu.ar

Recibido: 13 noviembre de 2015/ Modificado: 3 de diciembre de 2015 / Aceptado: 19 de junio de 2016

Para citar este artículo

Busso, A. (2016). Unasur en el escenario del multilateralismo latinoamericano: luces y sombras. OASIs, 24, 45-67.

DOI: http://dx.doi.org/10.18601/16577558.n24.04
} 
de esta forma, los principales desafíos que se presentan para el multilateralismo sudamericano en el nuevo siglo.

Palabas clave: UNASUR, Suramérica, multilateralismo, concertación política, condicionantes regionales, condicionantes extrarregionales

\section{UNASUR in the Latin American regional scene: lights and shadows}

\section{ABSTRACT}

Latin America, and South America in particular, faced different scenarios in the first decade of the century. Among the experienced changes, it is possible to highlight the emergence of the "post-hegemonic regionalism". In this context, new scenarios of cooperation between states in the region have emerged. This situation has reopened old debates and established new ones, mainly regarding the nature of these new integration schemes: whether they imply a new Latin American multilateralism; whether this sort of multilateralism has a distinctive feature and what impact do these processes have in relation to links with regional and extra-regional powers. In this article we intend to advance some reflections on the specific case of the Union of South American Nations (UNASUR) trying to answer the above enquiries. From this analysis, it may be noted that a singular practice was introduced: South American multilateralism. It is characterized, among other features, by constituting a process of policy consultation, or of multifunctional integration, in contrast to the traditional economic vision of the integration processes. However, it should also be mentioned that this process was not linear and has not been without difficulties. Thus, UNASUR has had periods of lights and shadows, all of which highlight the major challenges that arise for South American multilateralism in the new century.

Keywords: UnASUR, South America, multilateralism, political consultation, regional conditions, extra-regional conditions.

\section{INTRODUCCIÓN}

Latinoamérica, y Sudamérica en particular, afrontaron un escenario diferente en la primera década del siglo xxi. Conceptos como identidad latinoamericana, integración, concertación regional, autonomía política, entre otros, aparecieron mencionados e identificados como ejes destacados de las políticas exteriores de los países incluidos en el denominado "giro a la izquierda". En el ámbito sudamericano estas ideas permitieron abordar la compleja herencia de la hegemonía neoliberal de los años noventa y posicionar a la región como una "periferia desafiante" que discutía los criterios centrales del Consenso de Washington. Además, obtenía resultados exitosos logrando acciones "reparadoras" para aquellos sectores sociales excluidos como consecuencia de los efectos negativos del modelo de globalización mientras que, simultáneamente, se abordaban los problemas macroeconómicos que afectaban al conjunto de las sociedades nacionales. 
Sin embargo, esta recuperación de una visión latinoamericanista no implicó un camino lineal hacia su consolidación. El derrotero hacia una América Latina unida sobre la base de componentes identitarios comunes, fortalecida en la integración y la concertación con logros autonómicos en el manejo de los asuntos externos constituye un desafío aún vigente. Entre las razones que explican los escollos del camino existen cuestiones de orden regional tales como la persistencia de una heterogeneidad ideológica significativa entre países importantes para el devenir latinoamericano y otras de orden externo, tales como las presiones que ejercen el sector financiero transnacional, los sectores agroexportadores, los Estados centrales y los medios de comunicación hegemónicos, en la defensa de los intereses del modelo neoliberal.

Entre los cambios producidos en los primeros ańos del presente siglo se destaca la aparición del denominado "regionalismo poshegemónico". En ese marco surgieron nuevos escenarios de cooperación entre los Estados de la región tales como la Unión de Naciones del Sur (unasur), la Alianza Bolivariana para los Pueblos de Nuestra América (ALBA) y la Comunidad de Estados Latinoamericanos y Caribeños (CELAC). Esta situación reabrió viejos debates e instauró nuevos en torno a cuestiones tales como si estos procesos presentaban nuevas instancias de integración; si asistíamos a un nuevo multilateralismo latinoamericano; si dicho multilateralismo tenía características distintivas y qué impacto tenían estos procesos en relación con los vínculos con las potencias regionales y extrarregionales.
En este artículo pretendemos avanzar algunas reflexiones sobre el caso específico de la UNASUR, tratando de dar respuesta a los interrogantes antes mencionados. Para ello, haremos primero algunas aclaraciones de orden conceptual, posteriormente analizaremos el contexto político en el que surgió este proceso y finalizaremos mencionando sus principales desafíos.

\section{UN ACERCAMIENTO AL DEBATE CONCEPTUAL}

En América Latina, los debates sobre la integración regional, el multilateralismo, el regionalismo y las instancias de concertación suelen ser abordados de manera similar y, consecuentemente, las evaluaciones que se hacen sobre las experiencias desarrolladas en torno a los tres últimos conceptos suelen ser negativas. Una ajustada comprensión de las luces y sombras de la UNASUR requiere adentrarnos en el debate conceptual en torno a los conceptos recién mencionados.

En referencia a la integración regional, las conceptualizaciones clásicas, como la de Ernst Haas (1971, p. 6), la caracterizan como un proceso a través del cual los estados "se mezclan, confunden y fusionan voluntariamente con sus vecinos, de modo tal que pierden ciertos atributos fácticos de la soberanía, a la vez que adquieren nuevas técnicas para resolver conjuntamente sus conflictos".

Por su parte, Malamud y Schmitter (2006, p. 17) consideran que es necesario complementar esta definición subrayando que dichos procesos crean instituciones permanentes y pueden tomar decisiones vinculantes para 
todos sus miembros. Asimismo, mencionan otros elementos que contribuyen a tornar más probable la integración, pero no son su equivalente, a saber: el mayor flujo comercial, el fomento del contacto entre las élites, la facilitación de los encuentros o comunicaciones de las personas a través de las fronteras nacionales y la invención de símbolos que representan una identidad común. Además, Malamud establece cierta equiparación entre los conceptos de integración y regionalismo, mientras que subraya las diferencias entre este último y la regionalización. En ese marco, sostiene que la integración regional -en ocasiones denominada regionalismo- consiste en un proceso formalizado y conducido por el Estado.

En contraste, la regionalización -o aumento de la interdependencia intrarregional- es un proceso informal por el cual se incrementan los flujos de intercambio entre un conjunto de países territorialmente contiguos. Este proceso puede promover la integración formal que, a su vez, puede retroalimentarlo, pero su motor principal no reside en el Estado, sino en el mercado y, secundariamente, en la sociedad civil (2011, p. 220).

Finalmente, gran parte de la literatura destaca que los procesos de integración valoran la dimensión económica como su nota distintiva. Esta puede, a través de las distintas categorías señaladas en el clásico trabajo de Béla Balassa (1961), transitar por cuatro pasos que involucran desde la simple desaparición de aranceles comerciales hasta la coordinación de políticas macroeconómicas: a saber, área de libre comercio, unión aduanera, mercado común y unión económica.
Por otra parte, la "concertación política" está asociada al concepto de cooperación y, simultáneamente, hace alusión a instancias o mecanismos flexibles de diálogo político a nivel regional. Los principales antecedentes en Latinoamérica son el Grupo de Contadora, el Consenso de Cartagena y el Grupo de Río. Estos casos demuestran que desde inicios de los años ochenta y ante la parálisis que sufría la OEA como organismo internacional encargado de atender la agenda hemisférica, los gobiernos regionales decidieron potenciar espacios multilaterales con baja institucionalidad; que actuaban a nivel presidencial y ministerial; buscaban un consenso básico para atender un tema único (Contadora y el Consenso de Cartagena) o una agenda múltiple (Grupo de Río) y excluían a Estados Unidos.

En lo que refiere al multilateralismo, Robert Keohane (1990, p. 371) lo define como "la práctica de coordinar políticas nacionales en grupos de tres o más Estados”. Por su parte, John Ruggie (1992, p. 566) sostiene que "el multilateralismo refiere a la coordinación de las relaciones entre tres o más Estados de acuerdo con ciertos principios”. Tal como lo muestran estas definiciones, para que exista multilateralismo es ineludible contar con un mínimo de tres Estados que tengan la voluntad y la decisión de coordinar. Sin embargo, ambos conceptos son más flexibles en referencia a las temáticas sobre las cuales se aplicará la coordinación. Para el primero de los autores esta debe darse en referencia a las "políticas nacionales", mientras que para el segundo dicha coordinación gira en torno a las "relaciones" entre los Estados involucrados. 
En Sudamérica, a inicios del siglo XXI surgió, como consecuencia de las fuertes críticas a los resultados del regionalismo abierto y la aplicación de las políticas neoliberales de los ańos noventa, una nueva ola integracionista. En las descripciones de estos procesos se produce un entrecruzamiento de los conceptos mencionados hasta aquí y se suman otros. Si bien los análisis se insertan dentro de los estudios de integración, se los denomina "regionalismo posliberal o poshegemónico" (Sanahuja 2010, 2012; Legler, 2013) y, en menor medida, se los analiza como un "nuevo multilateralismo" (Legler, 2010; Oyarzún Serrano, 2010; Legler y Santa-Cruz, 2011), que también puede adjetivarse como posliberal o poshegemónico. Otros asocian a los nuevos procesos con la integración pero desde una perspectiva multidimensional (Hettne y Soderbaum, 2002; Frenkel, 2015).

La presencia de nuevos líderes políticos progresistas en América del Sur creó el ambiente propicio para el surgimiento del nuevo regionalismo no centrado en el tema del comercio y el relanzamiento de los procesos existentes dentro de esta nueva lógica. Este regionalismo se basó en el supuesto de que la liberalización de los flujos comerciales por sí sola no es capaz de promover el desarrollo, ni una agenda integrada para la construcción de la equidad social (Cienfuegos y Sanahuja, 2010).

Según Rios y Veiga (2007), esta ola de regionalismo fue llamada por algunos expertos como "posliberal" y establece la necesidad de desarrollo, la superación de la pobreza y la desigualdad, así como la incorporación de los grupos sociales que fueron excluidos de los modelos de integración liberales. A estas características Sanahuja agrega otras como: el retorno de la política a las relaciones exteriores; una agenda de desarrollo pos-Consenso de Washington; el Estado como actor central; la búsqueda de autonomía; la aparición de una renovada agenda de paz y seguridad; las carencias de la infraestructura regional; el énfasis en la seguridad energética; la promoción de una mayor participación de actores no estatales y la legitimación social de los procesos de integración (Sanahuja, 2009).

Desde otra perspectiva, Thomas Legler (2010) identifica simultáneamente este proceso como "multilateralismo" o "regionalismo posneoliberal” (Legler, 2013). En sus análisis destaca que estos procesos no implican que estemos asistiendo a experiencias de integración en tanto no existe cesión de soberanía. Además, identifica una serie de problemas propios del multilateralismo posneoliberal que impiden que se fortalezca la gobernanza regional, entre los que destaca: la tradición defensiva del multilateralismo (como forma de balancear el poder de Estados Unidos); falta de una identidad regional fuerte (región muy heterogénea, solo los une resistir a Estados Unidos); la competencia y superposición debido a la proliferación de espacios subregionales; el déficit de financiamiento que enfrentan estas nuevas instituciones (dependencia de poderes externos, ausencia de mayores compromisos por parte de los Estados nacionales); mentalidad de club (espacios multilaterales cerrados para las élites gubernamentales, sin inclusión de la sociedad civil); renuencia a delegar autoridad nacional (Legler, 2010, p. 13).

Las adjetivaciones sobre el regionalismo sudamericano del siglo XXI no incluyen solo 
la característica de posliberal. Algunos autores como Riggirozzi y Tussie (2012) lo definen como "regionalismo poshegemónico", subrayando el fin de una etapa de primacía de un único modelo de integración y el inicio de otra donde no hay recetas únicas para implementar. Señalan que los procesos actuales van más allá de una mera reacción a la crisis del neoliberalismo, y que son una manifestación expresa de la repolitización de la región que genera nuevas formas de hacer política y de construir proyectos regionales donde los Estados, los movimientos sociales y los líderes son los protagonistas del proceso.

Estos regionalismos superpuestos (Riggirozzi, 2010) tienen en común expresar estrategias alternativas para alcanzar crecimiento económico con justicia social; así como constituir una expresión de América Latina más politizada y recelosa del liderazgo político norteamericano. Son proyectos que revisan el modelo hegemónico de la década anterior y buscan alternativas acordes a sus realidades regionales. Resaltan su compromiso popular con la democracia y el nacionalismo, con mercados abiertos y políticas macroeconómicas heterodoxas, pero que no rompen con el capitalismo de mercado. Los gobiernos buscan reducir la dependencia del financiamiento externo y realizar una reconceptualización de la cooperación y armado de redes regionales (Grugel, Riggirozzi y Thirkell-White, 2008; Riggirozzi, 2010).

Finalmente, mencionamos los trabajos que incluyen en sus análisis sobre diferentes alternativas de integración y regionalismo a la "integración multidimensional" (Hettne y Soderbaum, 2002). Estos entienden por multidimensional a aquellos procesos de integración en donde no existe la preeminencia de un área en particular, sino que las agendas y temáticas de cooperación corresponden a una multiplicidad de áreas (económicas, políticas, sociales, educativas, culturales, de seguridad, etc.). Dicha diversificación, además, no solo implica una ampliación de las temáticas de la integración sino que también pretende evitar una jerarquización de las mismas. Así, Alejandro Frenkel (2015) argumenta que en el caso particular de Sudamérica, el carácter multidimensional adquiere un significado disruptivo, en tanto marca un contrapunto con los esquemas de regionalismo abierto, característicos de los ańos noventa, que se definen por una clara hegemonía de las agendas económico-comerciales. La no jerarquización de las áreas posibilita también que el pulso de los procesos de integración no dependa exclusivamente de los avances en un área particular, mitigando los cuellos de botella y, al mismo tiempo, generando incentivos para cooperar en múltiples temáticas.

En suma, los conceptos de "regionalismo posliberal y poshegemónico", "multilateralismo regional" e "integración multidimensional” reflejan diferencias con la integración tradicional y, simultáneamente, incluyen elementos comunes que los interrelacionan: critican la centralidad de la cuestión comercial que había orientado a las agrupaciones regionales anteriores en detrimento de las preocupaciones por el desarrollo y el bienestar social; y explicitan la inquietud por aumentar la cooperación política entre los países a los efectos de garantizar una mayor autonomía regional en el sistema internacional (Pasquarie- 
llo y Correa, 2015). Consecuentemente, para gran parte de los autores que han analizado este tema en Latinoamérica durante el siglo xxI, el "regionalismo" - y sus variantes- no es un equivalente a la integración tradicional. En este sentido se diferencian de la asimilación entre ambos conceptos realizada por Malamud (2011). Asimismo, el uso de los conceptos "multilateralismo regional" o "multilateralismo sudamericano" aparece, en ocasiones, como sinónimo de "regionalismo".

De este breve repaso podemos concluir que la UNASUR no puede ser considerada como un proceso de integración desde la perspectiva tradicional y, por tanto, tampoco puede ser evaluada como tal. Su trayectoria muestra que no se privilegiaron las variables comerciales, no se buscó una estructura institucional compleja y no se planteó concesión de soberanía por parte de los Estados miembros en función de crear un espacio supranacional. Tal como lo demuestra el devenir histórico, este espacio multilateral nace en la reunión de Cuzco en 2004 como Comunidad Suramericana de Naciones (CSN) con los objetivos de avanzar en: 1) la concertación y coordinación política y diplomática que afirme a la región como un factor diferenciado y dinámico en sus relaciones externas; 2) la profundización de la convergencia entre MERCosur, la Comunidad Andina y Chile a través del perfeccionamiento de la zona de libre comercio, apoyándose en lo pertinente en la Resolución 59 del XIII Consejo de Ministros de la Aladi del 18 de octubre de 2004; 3) la integración física, energética y de comunicaciones en Sudamérica sobre la base de la profundización de las experiencias bilaterales, regionales y subregionales existen- tes (Comunidad Suramericana de Naciones, 2004). Como se observa, en esta primera instancia se pensó en una "Comunidad" que actuase como un espacio de convergencia de los procesos de integración existentes, los cuales habían nacido bajo la impronta de la "clave comercial".

Sin embargo en 2006, durante la Cumbre en Cochabamba, se dio a conocer el informe titulado "Un nuevo modelo de integración en América del Sur" donde se argumentaba que "La construcción de un nuevo modelo de integración no puede estar basada únicamente en las relaciones comerciales, sobre todo cuando es sabido que la región admite modelos distintos, CAN, Mercosur, Chile [...] los países deberán buscar una integración más amplia, así como formas de cooperación política, social, cultural" (Comunidad Suramericana de Naciones, 2006). Como consecuencia de estas consideraciones, el pilar comercial desapareció y en 2007, durante la Primera Cumbre Energética Sudamericana en Isla Margarita, los mandatarios decidieron reemplazar la CSN por UNASUR.

Por tanto, el espacio multilateral fue definido como un proceso de integración interestatal, posneoliberal y multidimensional, con una agenda innovadora que desplaza de sus prioridades la cuestión comercial para encumbrar la concertación política, así como también la dimensión social, cultural y de infraestructura.

Como muestra esta enunciación, tanto en la letra como en la voluntad de sus creadores, la UNASUR se identifica con los conceptos de "regionalismo y multilateralismo posliberal y poshegemónico", "integración 
multidimensional” y “concertación”, pero se aleja de cualquier pretensión de convertirse en un proceso de integración tradicional.

Finalmente, corresponde señalar que las evaluaciones sobre el futuro y la consolidación del regionalismo posneoliberal y poshegemónico en el que se inserta la creación de UNASUR son dispares. Existen quienes tienen una visión muy optimista sobre el mismo (Riggirozzi y Tussie, 2012) y quienes son escépticos (Diamint, 2013; Legler y Santa Cruz, 2011; Sanahuja, 2010; Serbin, 2010; Malamud, 2011).

Los primeros destacan que este proceso de transformación tiene al menos cuatro componentes interconectados: construcción social de una nueva política regional; "engrosamiento" regional; protagonismo regional de la sociedad civil y capacidad de recuperación de la integración regional latinoamericana (Legler, 2013). Por su parte, los escépticos subrayan la persistencia de antiguas prácticas políticas y patrones institucionales, problemática que se aúna a la diversidad y fragmentación de las experiencias regionales. En primer lugar, se critica la noción de una nueva política regional, en tanto los esquemas institucionales creados en los últimos años, como ALBA, CELAC y UNASUR, se manifiestan como esquemas vacíos de un multilateralismo sustantivo debido a que los líderes se resisten a delegar verdadera autoridad. En todo caso, la nueva política regional se reduciría a un espacio interpresidencial. En segundo lugar, los escépticos cuestionan también si se ha producido un engrosamiento regional tanto en términos económicos como en relación con la formación de la identidad regional.
En efecto, dan cuenta de que los flujos comerciales intrarregionales han sido débiles y que la proliferación de estrategias regionales se ha debido más a decisiones presidencialistas que a demandas "desde abajo" fruto de una identificación popular con las nociones compartidas de América Latina o América del Sur. En tercer lugar, se sostiene que el regionalismo poshegemónico no está dando lugar a la potenciación de las redes de actores no estatales. En su lugar, las tendencias regionalistas reflejan un pronunciado déficit democrático. Por último, esta visión escéptica de la integración regional da cuenta de que lejos de observarse una unidad de propósito y compromiso entre los Estados que participan del regionalismo poshegemónico, los esquemas que predominan reflejan intereses puntuales de los Estados encargados de liderar dichos proyectos (Legler, 2013).

Estas diferencias nos obligan a ser cuidadosos a la hora de hablar de la UNASUR como un punto de inflexión y profundización del patrón regional, como vaticina parte de la literatura sobre la existencia de una tercera oleada de integración regional. De acuerdo con Botto (2015) los clivajes en cuanto al grado de novedad de este proceso se sustentan en las diferentes percepciones respecto de cuál es el carácter central de la integración. Si se priorizan las cuestiones comerciales, la UNASUR es solo un ejercicio de cooperación política (Bouzas, Motta-Vega y Ríos, 2007). En cambio, si lo que se destaca es la pretensión autonómica de la región por fuera de las cuestiones comerciales, incluyendo por primera vez la de defensa y seguridad (geopolítica), la UNASUR puede ser considerada como un 
proceso novedoso de integración profunda (no comercial) (Sanahuja, 2012).

\section{LA EXPERIENCIA NEOLIBERAL EN AMÉRICA LATINA Y EL SURGIMIENTO DEL MULTILATERALISMO POSHEGEMÓNICO}

En este apartado recorreremos, por un lado, las tendencias principales que explican el impacto del orden neoliberal sobre nuestra región y, por otro, las mudanzas del orden internacional a inicios del siglo xxi y las alternativas esgrimidas desde la región en pos de superar la crisis heredada de los años noventa y reivindicar el espacio latinoamericano entre las que se encuentra la UNASUR y otros procesos de regionalismo/multilateralismo posliberal y poshegemónico.

Un análisis contextualizado de lo acontecido en los últimos ańos nos remite a la etapa final de la Guerra Fría y el inicio de la posguerra fría. Este periodo de transición en el orden internacional estuvo marcado por la hegemonía neoliberal que encontró su expresión inicial en la llamada revolución neoconservadora liderada por Ronald Reagan y Margaret Thatcher. En ella se articularon una concepción política conservadora, una visión económica neoliberal que terminó consolidando el poder de las finanzas sobre la producción y una concepción de política exterior con fuertes componentes coercitivos. América Latina, y en especial Sudamérica, recibieron los primeros efectos negativos de esta mutación internacional cuando retornaban a la democracia. En este marco se convirtió en una región exportadora de capitales vía el pago de los servicios de su deuda que exigie- ron la banca privada y el FMI bajo el auspicio de la administración Reagan. Los intentos latinoamericanos de resistencia para abordar desde otra perspectiva los temas de la agenda internacional de entonces se canalizaron en la creación de instancias de concertación política (Contadora, Consenso de Cartagena y el Grupo de Río) sin la presencia de Estados Unidos. Si bien no podemos desconocer algunos éxitos políticos de estos procesos, también debemos remarcar la resistencia de Washington a cada una de estas instancias y su capacidad para frenar la creación del club de deudores propuesto en Cartagena (Busso, 2014).

La década de los noventa se convertiría en el escenario de afirmación de la hegemonía neoliberal. La caída de muro de Berlín en 1989 y la implosión de la uRss en 1991 consolidaron la condición de Estados Unidos como ganador de la Guerra Fría. Bajo los argumentos del fin de la historia (Fukuyama, 1989), los supuestos políticos y económicos del neoliberalismo fueron universalizados. Consecuentemente, las condicionalidades globales sobre el mundo en desarrollo permitían una única alternativa: democracia formal liberal articulada con economía de mercado y procesos de integración basados en el regionalismo abierto. La acción del Estado debía ser mínima mientras que la distribución de la riqueza quedaba en manos del mercado (Busso, 2014).

Lo más notorio es que la consolidación del dominio neoliberal se produce bajo las administraciones Clinton y Blair quienes, en el marco discursivo "de la tercera vía" que caracterizó a la socialdemocracia de la época, aplicaron una versión más light del modelo (la tarea más dura había sido realizada por el 
tándem neoconservador), pero habilitaron el afianzamiento de un nuevo bloque de poder constituido por los sectores financieros, los grandes sectores agroexportadores -especialmente los ligados a la soja- y los medios de comunicación privados. En este marco, la mayoría socialdemócrata se convertía en portavoz de la globalización (Sader, 2009).

En el ámbito de la acción externa latinoamericana, las dos consecuencias más significativas de lo acontecido en los años noventa fueron: la instauración de una etapa de unipolaridad de Estados Unidos que se presentó bajo el formato de una hegemonía benevolente, y la consolidación de un escenario de homogeneidad de las políticas exteriores y económicas de América Latina caracterizadas, aunque con diferentes intensidades, por el alineamiento / acoplamiento ${ }^{1}$ con Washington.

El nuevo siglo trajo consigo un panorama diferente en las relaciones internacionales latinoamericanas, donde se distinguen dos etapas. Una inicial, que atraviesa la primera década, y una segunda fase que se inicia a finales de la misma y se prolonga hasta nuestros días.

La nota distintiva de los primeros diez ańos del siglo estuvo relacionada con la disminución de la densidad de los vínculos entre Estados Unidos y América Latina, en general, y América del Sur en particular, y un incremento de la autonomía y la visibilidad internacional de la región donde resaltaba el intento de la construcción de un proceso identitario común y la búsqueda de renovadas concepciones de integración / concertación.

Como suele ocurrir con los procesos políticos, las causas de este cambio fueron múltiples. Si posamos la mirada en los asuntos de orden sistémico, se destaca la desatención hacia el sur del continente por parte del Gobierno de George W. Bush desde 2001, el cual se encontraba abocado a librar la guerra contra el terrorismo internacional, que privilegiaba otras regiones del planeta. En ese marco, Latinoamérica no constituía una amenaza significativa. Por otra parte, la excesiva militarización de la política exterior estadounidense durante esa etapa generó rechazos a nivel global y también en varios países sudamericanos. Esta tendencia se agudizó cuando la administración Bush decidió, en 2003, ir a la guerra contra Irak y se consolidó con el estallido de la crisis de 2008, tras la cual se acentuó la crisis relativa de hegemonía estadounidense y la disminución de la influencia política y económica de Washington sobre la región ${ }^{2}$.

Russell y Tokatlián (2008) entienden que el acoplamiento se caracteriza por un plegamiento a los intereses estratégicos y vitales de Estados Unidos, tanto en el ámbito global como regional.

2 Entre los acontecimientos políticos más notorios se destacan el rol de México y Chile, que se desempeñaban como miembros no permanentes del Consejo de Seguridad y anticiparon que no votarían a favor en caso de que el Gobierno republicano presentase un proyecto de resolución destinado a conseguir el apoyo de la Organización para iniciar la guerra contra Sadam Hussein. Esta actitud de América Latina es subrayada como uno de los principales ejemplos de una autonomía regional creciente. A nivel económico-comercial, es posible mencionar que la participación de Estados Unidos en las exportaciones sudamericanas se contrajo del 30 al $18 \%$ entre 2000 y 2011 , y las importaciones sudamericanas de Estados Unidos cayeron del 55 al 30 \% (Fernández, 2013). 
Paralelamente, en 2001 China ingresaba a la Organización Mundial del Comercio (OMC) y comenzaba a potenciar su proyección económica global. Su presencia en Latinoamérica se incrementó en un tiempo breve generando un cambio estructural en la matriz de comercio exterior de la región. Como lo muestran los datos de la Comisión Económica para América Latina y el Caribe (CEPAL) (2011 y 2012) el país asiático se transformó en el primer mercado para las ventas de Brasil, Perú y Chile, y en el segundo para Argentina, Colombia, Paraguay, Uruguay y Venezuela, desplazando al mercado estadounidense en las plazas sudamericanas. Un repaso de las relaciones comerciales establecidas por China con la región muestra que las mismas tienen un carácter pragmático que le ha permitido a Beijín obtener las materias primas (alimentos, minerales, petróleo) que necesita, e incrementar los vínculos económicos independientemente del perfil ideológico de su contraparte. Este panorama se repite en la política de inversiones del gigante asiático hacia la región, las cuales son acompañadas con un lento pero constante componente político. Así, el Gobierno de China publicó en 2008 el Libro Blanco para América Latina y el Caribe, estableciendo las principales líneas de acción política, económica, militar y cultural hacia la región; en el mismo año cambió su condición de país observador a país donante del Banco Interamericano de Desarrollo (BID); coincidió en numerosas ocasiones con los países de América del Sur en las votaciones en la ONU, y creó un grupo de Apoyo por Malvinas.

Sin embargo, la nota distintiva de esta relación es la diferencia en la modalidad de los vínculos que ha establecido China con América Latina en comparación con los sostenidos por los Estados occidentales y los organismos multilaterales de crédito. En este marco, la decisión de China de optar inicialmente por una estrategia que no incluya condicionalidades previas vinculadas al tipo de modelo de desarrollo, a la organización del sistema político o a las acciones de política exterior en general como requisito previo a los acuerdos de comercio e inversión, la ha diferenciado sustancialmente de los poderes occidentales. Si bien esta situación puede ser temporal, lo cierto es que resultó de gran utilidad para la aceleración de los contactos con Latinoamérica y fue, además, un instrumento que colaboró para que la región enfrentase la crisis económica-financiera de 2008 con menor fragilidad. Todo esto sin desconocer los desafíos que, en términos de desarrollo, conlleva el vínculo económico comercial con China, entre los que se destaca la reprimarización y la bilateralidad con la que establece los vínculos, promoviendo indirectamente una fragmentación en la cohesión regional.

En esta atmósfera de cambios en la distribución del poder mundial también se inscriben otros de menor envergadura, pero no por ello menos importantes para los análisis sobre el devenir internacional realizados desde el sur. Uno de ellos es la acelerada recomposición de la Federación Rusa que, en veinte ańos, pasó de ser el principal referente de la implosión de la URSS a recobrar la condición de potencia regional. Dicha tendencia se trasladó al vínculo entre Moscú y los países latinoamericanos, dando señales de un incremento en su densidad desde principios de 
la década del 2000, la cual se manifiesta en las dimensiones comerciales, de inversión y político-diplomáticas.

Otro cambio para tener en cuenta en la primera década del siglo fue el rol de Brasil como actor político y económico en América del Sur y el consecuente aumento de su proyección internacional durante el Gobierno de Lula da Silva. Bajo este periodo Brasilia incrementó la búsqueda de autonomía política, económica y estratégica. Su proyección como global player se estructuró a partir de tres ejes: el soft-balancing (Flemes, 2010; Giaccaglia, 2010), la diversificación de vínculos y la unidad colectiva. En este último aspecto, la UNASUR se transformó en una pieza nodal de su estrategia, dado que a través de la misma se intentó alcanzar un objetivo histórico de la política exterior de Brasil, como ha sido cohesionar a Sudamérica bajo su liderazgo. Sin embargo, es importante subrayar que Brasil no planteó un único y sofisticado modelo de integración, sino que buscó crear una plataforma para el diálogo político y la cooperación. La asimetría que caracteriza las relaciones entre Brasil y sus vecinos, y el mantenimiento de los principios de soberanía explican por qué no se propone la creación de instituciones supranacionales, lo cual nunca estuvo en la agenda (Gratius y Saraiva, 2013). En este marco, una propuesta de integración light le resultó funcional tanto para su proyección regional como global.

En suma, las tendencias hacia el cambio acaecidas en el orden internacional en la primera década del siglo XXI encuentran su principal manifestación en el incremento del poder relativo de un conjunto de potencias emergentes (BRIC/ BRICS y otras no incluidas en este grupo) que representan, en términos de Fareed Zakaria (2008) "el ascenso de los otros". Estas tendencias no conllevan una implosión ni una caída abrupta del poder de Estados Unidos, pero sí una distribución de poder distinta para el orden internacional en ciernes que, en el caso de América Latina en general, pero muy especialmente en Sudamérica, se materializó en el periodo 2000-2011 en una búsqueda de mayor autonomía política y económica para lo cual la concertación / integración regional y la diversificación de vínculos fueron centrales. Todo este proceso se enmarcó en una etapa de precios muy altos para los commodities, situación que fue extremadamente favorable para las arcas de los Estados latinoamericanos.

Por otra parte, si reflexionamos sobre lo acontecido a nivel regional en este periodo, sin dudas, el aspecto más relevante es la crisis neoliberal y su impacto sobre la región. En ese escenario, Sudamérica adquiere una dinámica especial. Un conjunto de países fueron partícipes del llamado "giro a la izquierda", que con características e intensidades diferentes, compartieron el criterio sobre la necesidad de modificar varias de las políticas aplicadas en los años noventa. Así, cuestiones como una mayor participación del Estado como regulador de la economía; la aplicación de políticas distributivas; el desarrollo de los mercados internos; la opción por políticas activas para afrontar los efectos de la crisis desatada a partir de 2008; la jerarquización de las políticas de integración regionales y subregionales, y la búsqueda de una acción externa más autónoma aparecen como denominadores comunes. 
En este escenario Sudamérica impulsa, con Venezuela como líder, la creación del ALBA y, a propuesta de Brasil, la UNASUR. Esta última se destaca por implementar mecanismos de convivencia exitosos no solo entre los países cuyos giros ideológicos han sido más agudos (Bolivia, Ecuador, Venezuela) y los más moderados (Argentina, Brasil, Chile -con Lagos y Bachelet- Paraguay -hasta la caída de Lugo-, Uruguay) sino también entre todos ellos y los Estados que optaron por continuar o retomar el modelo económico neoliberal como Colombia, Perú y Chile. En este marco se enfatizan las acciones conjuntas fruto de los lazos creados por UNASUR ante hechos como: los intentos secesionistas de los estados de la medialuna en Bolivia (2008); la crisis por el acuerdo para el uso de bases colombianas por parte de las Fuerzas Armadas estadounidenses (2009), el golpe de Estado en Honduras (2009), el intento de desestabilización política en Ecuador (2010) y la destitución de Fernando Lugo en Paraguay (2012). A lo anterior se le deben sumar también las acciones conciliatorias de otras instancias de concertación como el Grupo de Río en ocasión de la crisis entre Colombia y Ecuador por el ataque aéreo colombiano a un campamento de las FARC en territorio ecuatoriano (2008).

Las posturas a favor de la consolidación de los vínculos subregionales de los distintos gobiernos provinieron de bases diversas y fluctuaron desde los argumentos ideológico que rescatan el pensamiento BolivarianoSanmartiniano hasta los que se sumaron por cuestiones de pragmatismo ligadas, fundamentalmente, a que la agenda regional y las condiciones complejas del orden internacional eran mejor abordadas desde una perspectiva cooperativa y en un entorno subregional menos conflictivo. Esto no implicó la ausencia de problemas y enfrentamientos, pero sí la existencia de escenarios que han ido avanzando desde la tensión hacia un mayor entendimiento ${ }^{3}$.

Además, desde una mirada políticosociológica, el contexto de la crisis neoliberal impactó sobre la necesidad de pensar en términos de identidad nacional y regional. Si consideramos con Ortiz que el problema de la identidad surge cuando aparece "una presunta falta de correspondencia en forma de expectativas defraudadas entre el sujeto (y entre los sujetos) y el contexto" (Ortiz, 2007, p. 40) podemos inferir que, en la atmósfera de crisis social, política y económica que vivieron varios de los países sudamericanos a finales de los años noventa e inicios del presente siglo, las expectativas de los distintos actores sociales y políticos no encontraban respuestas, lo que incrementó fuertemente las demandas de cambios.

\footnotetext{
3 Muestra de ello son las relaciones entre Venezuela y Colombia, y los vínculos entre este último país y Ecuador a partir del Gobierno de Santos; la disminución relativa de las tensiones entre Argentina y Uruguay con posterioridad a la crisis por las pasteras; la disposición a respetar mecanismos de solución de controversias cuando la diplomacia bilateral no alcanza un acuerdo, como ocurrió entre Perú y Chile en el debate sobre la fijación de sus límites marítimos en la Corte de La Haya, y la creación de ambientes positivos para la solución de problemas de larga data como el generado en las negociaciones entre el Gobierno colombiano y las FARC.
} 
En ese marco, Moreira, Raus y Gómez Leyton (2008) afirman que los gobiernos sudamericanos del giro a la izquierda se plantearon la necesidad de construir nuevas identidades sociales. Así, trataron de dar respuesta tanto a la fragmentación de las identidades sociales clásicas de la etapa populista-desarrollista como a la emergencia de múltiples identidades. Algunas de ellas se definieron socioculturalmente, a partir de ser portadoras de demandas extraeconómicas. Otras lo hicieron desde lo socioeconómico, pero mostrando la regresividad y desarticulación de este aspecto a partir de los ańos noventa. Consecuentemente, estos sectores se expresaron desde la exclusión, más que desde la inclusión, al mercado de trabajo. Asimismo, destacan que esta diversidad de sujetos sociales dificultó el armado del discurso político de los nuevos gobiernos y, en numerosas ocasiones, generó tensiones entre el Estado y la sociedad, lo que se canalizó en situaciones de ingobernabilidad. La presencia de escenarios similares a nivel regional condujo a los gobiernos a buscar elementos en común que contribuyeran a la idea de "la patria grande", con la que se intentaba construir una identidad regional basada en la historia y los problemas comunes.

Todo este activismo multilateral subregional se ha llevado adelante sin la participación de Washington, y los hechos confirman esta tendencia ya que desde los procesos de integración nacidos en los ańos noventa como MERCOSUR o aggiornados en esos ańos, como la Comunidad Andina, se avanzó hacia la unasur, el alba y la Celac. Dicho multilateralismo de baja institucionalidad, pero de intensa actividad en los primeros años del siglo XXI, se contrapuso a las preferencias de Washington por el bilateralismo como instrumento para abordar los temas de la agenda regional. Por otra parte, los diversos espacios multilaterales hemisféricos liderados por Washington perdieron dinamismo. Esta situación se reflejó, entre otras, en la OEA, las Reuniones Hemisféricas de Ministros de Defensa, el fracaso del proyecto del ALCA y, en el caso de la Cumbre de las Américas, se mostró que temas que antes eran claramente evitados por los presidentes de la región para no confrontar con Estados Unidos, comenzaron a plantearse abiertamente, incluso por parte de los países identificados -económica y políticamente- con Washington. Los reclamos de varios presidentes latinoamericanos a Obama en la reunión de Cartagena de Indias para la incorporación de Cuba a este sistema de cumbres (finalmente concretada en la cumbre de Panamá de 2015) y los planteos sobre la necesidad de iniciar un debate vinculado a nuevas modalidades de lucha contra el crimen transnacional, especialmente contra el narcotráfico, son ejemplo de ello (Busso, 2014).

En este marco de relaciones regionales positivas resulta, no obstante, necesario recalcar que la realidad latinoamericana en general y la sudamericana en particular no fue ideológicamente homogénea. Como ya lo apuntamos, existieron distintas dinámicas en el giro a la izquierda y, también, países que no mudaron y se mantuvieron bajo la égida del neoliberalismo. En palabras de Comini y Frenkel, existieron dos alternativas de vinculación con la región y el mundo implementadas por los Estados sudamericanos: 
Por un lado el modelo de inserción internacional poligámico el cual se fundamenta en una estrategia de transversalidad que prioriza el mercado internacional e implica negociaciones simultáneas con actores de los niveles global, hemisférico y regional. Esta estrategia no descarta la posibilidad de embarcarse en procesos de integración con otros países, siempre y cuando estos no generen compromisos que impidan avanzar, de forma individual, en la configuración de acuerdos con terceros. [...] Por otro lado, el modelo concéntrico que se sustenta en un esquema estado-céntrico, basado en una estrategia que prioriza los mercados regionales. Quienes asumen esta lógica de inserción internacional lo hacen partiendo de la premisa de que ellos, por sí solos, cuentan con márgenes reducidos de negociación con actores extrarregionales y con menores alternativas para imponer sus intereses. En ese marco, afirman la necesidad de definir espacios de consenso -no necesariamente comerciales- con los países vecinos y, una vez establecidos aquellos, de negociar en forma conjunta acuerdos de integración con terceros (Comini y Frenkel, 2014, p. 61).

Sin embargo, más allá de estas diferencias, lo notorio es que América del Sur avanzó hacia una orientación autonómica. Eso se debe, a nuestro entender, a la presencia simultánea de liderazgos presidenciales como los de Chávez, Lula y Kirchner, lo cual permitió imprimir una lógica política predominante en la región convirtiendo el giro a la izquierda en la nota distintiva del "cambio de época"; y, más significativo aún, logrando que el resto del mundo también percibiera el proceso político de Sudamérica en esa dirección. Esta tendencia fundacional y su impacto global se fortalecieron con la CSN primero y la UNASUR después, y también a partir de 2006 con la llegada de
Evo Morales y Rafael Correa a los gobiernos de Bolivia y Ecuador. Por otra parte, también coadyuvó el hecho de que presidentes que habían optado por no alterar significativamente la orientación económica de sus países, como el caso de Mitchell Bachelet durante su primer Gobierno, decidieron apoyar fuertemente las acciones políticas de UNASUR, fortaleciendo el proceso en cuestión. En síntesis, la idea de una Sudamérica que abrevaba de su identidad latinoamericana, solucionaba sus diferencias en el marco de la concertación regional y formulaba políticas exteriores autónomas tomó cuerpo en la arena internacional de los inicios del siglo XXI.

\section{LA SEGUNDA DÉCADA DEL SIGLO XXI: CAMBIOS INTERNACIONALES, DESAFÍOS REGIONALES Y CONSECUENCIAS SOBRE EL MULTILATERALISMO SUDAMERICANO}

La llegada de la segunda década del siglo trajo consigo un conjunto de desafíos para la consolidación del proceso de cambios iniciado en Latinoamérica. Esos desafíos se producen como fruto de nuevas mudanzas a nivel internacional, y la aparición de dificultades políticas y económicas de distinta intensidad en los Estados regionales.

Pasando revista a la situación internacional visualizamos, en primer lugar, que la política externa de Obama hacia la región en su segundo mandato es más activa. Esta actividad no solo implica mayor presencia de Washington, sino también una conjugación de estrategias (positivas y negativas) que contribuyen a acentuar la heterogeneidad político-ideológica regional e incrementar las disputas domésticas 
en algunos países de la región, afectando la posibilidad de que Latinoamérica consolide las políticas iniciadas en la década pasada. Así, la Casa Blanca modificó su política hacia Cuba y avaló las negociaciones de paz entre el Gobierno colombiano y las FARC, coincidiendo con las demandas latinoamericanas pero, por otra parte, restringió las posibilidades de diálogo con el Gobierno venezolano mientras apoya oficialmente a través de sus embajadas y de la Agencia de los Estados Unidos para el Desarrollo Internacional (USAID, por sus siglas en inglés), y en forma privada vía distintas ONG, las acciones de la oposición favoreciendo el distanciamiento entre el Gobierno y la sociedad $^{4}$; restringió el apoyo que el Ejecutivo estadounidense le brindó inicialmente a Argentina en la disputa con los holdouts; mantiene una relación moderada hacia Brasil y plantea un acercamiento a los países de la Alianza del Pacífico como reconocimiento a la modalidad de integración neoliberal propuesta por esas naciones ${ }^{5}$. Esta última estrategia tiene varias implicancias: mostrar un MERCOSUR en crisis; detener la consolidación de la UNASUR; afectar el desarrollo de la CELAC y, globalmente, avanzar con la implementación del Acuerdo de Asociación Trans-Pacífico (TPP) que, cuando logre su ratificación, se convertirá en el mayor bloque comercial del mundo. Tanto por razones político-económicas como por geografía, este proceso incluye a Chile, Perú y México, y está abierto a otros que deseen orientar su economía siguiendo la tradición neoliberal, lo que ha generado quiebres al interior de las élites del resto de América Latina.

En este contexto, algunos medios de comunicación tienden a subrayar ciertos indicadores de crecimien to económico de los países de la Alianza del Pacífico como los únicos assets para tener en cuenta. Así, dado el mayor crecimiento anual del PBI de tales economías ${ }^{6}$, infieren una interpretación política que pone fin a los éxitos logrados en el marco del giro a la izquierda e inauguran una nueva etapa en la región basada en la recuperación de muchos de los criterios seguidos en los años noventa. En sus análisis han alabado a México, Colombia y Perú, mientras critican constantemente las condiciones democráticas y económicas de Argentina, Bolivia, Ecuador y Venezuela. En los últimos meses, invocando los problemas

4 Como referencia de las acciones de las ong estadounidenses ver: CEPRID (2009), Eje TV (2013) y Laborde (2014).

5 De acuerdo con las declaraciones del portavoz de la Casa Blanca: "The United States applauds the progress the Pacific Alliance has made to integrate its markets and the strong economic policies pursued by its members as examples for Latin America. We intend to use our observer status to recognize and support these achievements and to advance the values we share with the member states, including commitments to expanding free markets, reducing inequality, opening trade, and welcoming foreign investment. The United States already enjoys strong and productive economic relations and a free trade agreement with each Pacific Alliance member country. Our participation as an observer is a natural extension of those existing economic relationships and our cooperative efforts under the Trans-Pacific Partnership with Alliance members Chile, Peru, and Mexico".

6 Véase Datos del Banco Mundial, http://data.worldbank.org/data-catalog/world-development-indicators 
de corrupción, han incrementado las críticas a Brasil y han puesto en duda a Chile ${ }^{7}$.

Saliendo de nuestro continente, otras variaciones internacionales que han aparecido en los últimos años tienen que ver con China, la Federación Rusa, los BRICs y la economía internacional. Numerosos análisis sobre la República Popular subrayan que la disminución de sus tasas de crecimiento podría afectar la política de inversiones que el gigante asiático se ha comprometido a realizar en Latinoamérica y, simultáneamente, disminuir sus importaciones desde nuestra región. Por otra parte, si bien el Gobierno de Putin ha mantenido su postura de acercamiento a la región en términos políticos y económicos, también es cierto que las sanciones aplicadas por Estados Unidos y la Unión Europea por la crisis en Ucrania tuvieron un doble efecto: por una parte, fomentaron que Moscú favoreciera la compra de materias primas desde América Latina pero, por la otra, su economía y el valor de su moneda se vieron fuertemente afectados. Si a esta situación le sumamos el estancamiento de la economía brasileña, el resultado es una desaceleración de la dinámica económica de los BRICs, y una parálisis -al menos temporal- del proyecto político para crear un orden internacional más equitativo y no hegemónico liderado por los poderes emergentes. Finalmente, en los últimos tres años, la baja de los precios internacionales de los commodities ha disminuido notoriamente los ingresos por exportaciones de la región y, muy especialmente, el de las economías ligadas al petróleo, los minerales y la soja.

Este escenario de cambios impacta a su vez en la dinámica del regionalismo sudamericano. La conformación de la Alianza del Pacífico en 2011 afectó el ámbito regional $y$, para varios gobiernos, fue un planteo destinado a desafiar al MERCosur y la UNASUR. Según Comini y Frenkel (2014, p. 58), la polarización entre quienes pronostican el fin del sueño de la comunidad sudamericana y aquellos que reivindican la alternativa regional como espacio natural de comunión para la edificación de bienes comunes se ha visto notablemente acrecentada en los últimos tiempos. El pesimismo de los primeros adquirió impulso a la hora de analizar los resultados y las potencialidades del esquema de UNASUR. Tal como lo señalamos, se ha exaltado la idea de que la nueva Alianza representa el camino

A modo de ejemplo, Oppenheimer señala que de acuerdo con datos de la CEPAL, durante 2012 los cuatro países miembros de la Alianza del Pacífico tuvieron un índice de crecimiento combinado de $5 \%$, mientras que los países del Mercosur crecieron a una tasa promedio del 2,9\%; que el intercambio comercial entre los primeros creció 1,3\% mientras que entre los miembros del Mercosur cayó un 9,4\%. La conclusión política inmediata que cierra el análisis indica que "La Alianza del Pacífico [...] se está consolidando como un bloque económico con enorme potencial, mientras que el Mercosur, el mercado común constituido por Brasil, la Argentina, Venezuela, Uruguay y Paraguay- [...] se está convirtiendo en un comité político [...] cada vez más debilitado por disputas internas [...] En Cali, [los Estados que integran la Alianza] se presentaron como un grupo de países estables que respetan la democracia y el Estado de derecho y que, por tanto, ofrecen oportunidades de inversión mucho mejores que Venezuela, Argentina y otros países populistas que expropian empresas extranjeras a su capricho" (Oppenheimer, 2013). 
correcto hacia el éxito y se ha subrayado la capacidad disruptiva que podría tener sobre esquemas de integración como el Mercosur ${ }^{8}$, el cual aparecería señalado como un modelo y una estrategia económica desacoplada de los nuevos tiempos (Gualdoni y Robossio, 2014). Sin embargo, estas evaluaciones sobredimensionan el grado de innovación en materia de integración económica y los efectos de la Alianza del Pacífico, acuerdo que se construye con base en la red de tratados de libre comercio preexistente entre los países miembros. Esto nos permite inferir que las lecturas negativas sobre el devenir autonómico y neodesarrollista de Sudamérica se han incrementado no solo por la aparición de la Alianza, sino también por razones de orden político. En esa dirección, el presidente Correa (2013) expresó que "hay una restauración conservadora en la región para contrarrestar [...] la UNASUR" y que "la Alianza del Pacífico es [...] no querer crear una gran nación sudamericana sino tan solo un gran mercado".

Otra modificación que afecta la dinámica política iniciada en Sudamérica en la década pasada es la ausencia y pérdida de los liderazgos fundacionales. Néstor Kirchner muere en octubre de 2010, Lula da Silva finaliza su mandato en el mismo año y Hugo Chávez fallece en 2013. La desaparición de estas figuras alteró la interacción política que había dinamizado al bloque sudamericano en sus primeros ańos, especialmente la UNASUR. A este proceso se sumaría, en junio de 2012, la destitución-por medio de lo que se denominó un "neogolpe de Estado" - del presidente de Paraguay, Fernando Lugo, quien sería remplazado por su vicepresidente, Federico Franco. A pesar de que Lugo no había ejercido un liderazgo regional, sí había contribuido a la cohesión del grupo de países que representaban el modelo de integración concéntrico. En la misma dirección, la muerte de Kirchner trajo como consecuencia el debilitamiento de la Secretaría General de la UnASUR que había sido central para recomponer la relación bilateral entre Colombia y Venezuela, y actuar ante el amotinamiento policial en Ecuador en 2010, considerado un intento de golpe de Estado contra Correa. En el presente, la elección como Secretario General del expresidente colombiano Ernesto Samper, abre una posibilidad de reactivación de la Unión en tanto, después de una etapa de transición, el nuevo Secretario cumple con el requisito de ser un expresidente con capacidades y experiencia de negociación, tal como se había acordado en el marco de la institución?

Un tercer cambio está ligado a la evolución de los escenarios domésticos de varios de los países que lideraron el "giro a la izquierda". En líneas generales, la situación económica es

\footnotetext{
8 Ante el lanzamiento de la Alianza del Pacífico, Paraguay y Uruguay se sumaron como miembros observadores generando nuevos desacuerdos al interior de la UNASUR y tensiones en el MERCosur. Por esa razón, este último decidió designar a un Alto Representante para el cumplimiento de dicha función en representación del conjunto.

9 Esta evaluación optimista fue sostenida por el doctor Marco Aurelio Gracia en el marco de la conferencia de cierre pronunciada en la XII Semana de Relaçôes Internacionais da UNESP "Visões do Sul: Crise e Transformaçôes do Sistema internacional”, Franca (Brasil), 15 de agosto de 2014.
} 
menos prometedora desde 2012, y esto transcurre en un escenario donde la capacidad para movilizar amplios sectores de la sociedad civil con el fin de establecer nuevas y más profundas modificaciones, tanto en el ámbito de la economía como en materia de política exterior, es menor que hace una década. En ese sentido, el estancamiento del crecimiento de las economías de Brasil y Argentina, la crisis política y económica en Venezuela a partir de la muerte de Chávez y las dificultades políticas del Gobierno de Correa son señales de que los países del "giro a la izquierda" transitan en nuestros días un escenario de crisis. En este marco, el desempeño económico positivo de otras naciones de igual signo político, como los casos de Ecuador y Bolivia, no alcanza a revertir esa percepción negativa.

A pesar de todos estos desafíos, entre 2013 y finales de 2014, las urnas volvieron a darle apoyo a los gobiernos progresistas de la región. Los triunfos fueron muy contundentes en los casos de Rafael Correa, Michel Bachelet, Evo Morales y Tabaré Vásquez, y más acotados en el caso de Nicolás Maduro y Dilma Rousseff. Desde una perspectiva latinoamericana, esta tendencia se vio reforzada por la reelección del Frente Farabundo Martí en El Salvador. Sin embargo, en breve varios de estos gobiernos volvieron a enfrentar desafíos que afectaron su credibilidad y aumentaron los desacuerdos con la oposición política, los medios de comunicación y algunos actores internacionales. Con diversos argumentos Argentina, Brasil, Venezuela e, incluso, Chile han transitado o transitan situaciones de este tipo. En algunos casos este proceso es acompañado por el nacimiento de nuevos partidos autodefinidos como una nueva derecha democrática -no heredera de los autoritarismos, sino neoliberal por opción ideológica-, que se plantean como objetivo convertirse en opciones electorales que, más allá del grado de veracidad que no podemos constatar, prometen un cambio sin abandonar algunos de los logros alcanzados en la década pasada ${ }^{10}$. Sin embargo, el repaso de sus propuestas muestra una menor inclinación hacia la integración regional, una ausencia de referencias a la UNASUR como espacio de concertación política y una mayor predisposición a recuperar políticas de consenso con Washington y Europa bajo patrones de la experiencia neoliberal.

Por otra parte, los gobiernos del "giro a la izquierda”, aún en funciones, se encuentran ensimismados en sus propias y complejas agendas domésticas y han descuidado el regionalismo/multilateralismo poshegemónico. En ese marco, las acciones de la UNASUR han tenido resultados dispares. Manifestaron su agrado en el proceso de recomposición de vínculos entre Estados Unidos y Cuba; intentaron colaborar sin éxito en las profundas diferencias existentes entre el Gobierno y la oposición en Venezuela, y recuperaron activismo cuando a través de los presidentes protempore de

10 Esta postura lleva a varios autores a definir a estos nuevos partidos políticos no solo como una nueva derecha democrática, sino también como posneoliberal. Sin embargo, el hecho de que incluyan en su seno a buena parte de los sectores conservadores tradicionales y que sus propuestas de conservar los logros alcanzados no sean explicitadas con claridad, nos conduce a caracterizarlas como democráticas y liberales. 
la CELAC y la UNASUR primero, y luego con las reuniones propuestas por los cancilleres de Brasil y Argentina, se intentó encauzar la crisis fronteriza acontecida entre Venezuela y Colombia en 2015.

\section{CONCLUSIONES}

En el marco de la llamada tercera ola de integración acontecida en Latinoamérica se destaca la creación de la UNASUR. En términos conceptuales es preciso señalar que la misma debe ser entendida como un proceso de concertación política, regionalismo/ multilateralismo o, en su defecto, una integración multifuncional, descartándose su evaluación desde los paradigmas tradicionales economicistas de los procesos de integración. A nuestro entender, las nociones de concertación y regionalismo/multilateralismo son las que mejor la explican.

Conforme lo analizado, la UNASUR nace como consecuencia de los efectos negativos de la crisis neoliberal que transitaron varios de los países sudamericanos sobre finales de los años noventa e inicios del nuevo siglo, y que pusieron en cuestión el paradigma del regionalismo abierto, hegemónico durante las últimas dos décadas del siglo xx. En ese marco se potencian dos situaciones simultáneas: la aparición del metafóricamente llamado "giro a la izquierda" y la pérdida relativa de la influencia estadounidense en la región. De ahí su ubicación dentro del fenómeno del regionalismo/ multilateralismo posneoliberal y poshegemónico.

Como hemos señalado, el devenir de la UNASUR no fue lineal ni estuvo exento de di- ficultades. Esta ha transitado un quinquenio de luces y otro de sombras. Mientras que en el primero la convergencia ideológica, el crecimiento económico armónico y los fuertes liderazgos permitieron la creación y consolidación de este espacio, reconociéndose sus principales éxitos en la preservación de la institucionalidad democrática en la región; en el segundo, las heterogeneidades crecientes y el ensimismamiento de varios de los gobiernos otrora líderes del proceso, y en particular la irrupción en la región sudamericana de esquemas de integración alternativos basados en la tradición liberal, como la Alianza del Pacífico, erosionaron la relativa cohesión alcanzada por el subsistema sudamericano, materializada en la UNASUR. Conforme ello, si bien no podemos argumentar que a través de esta se haya consolidado una nueva identidad sudamericana, sí podemos sostener que se avanzó en esa dirección y que se incrementaron los vínculos políticos entre los países miembros como nunca antes. Además, durante el primer quinquenio la UNASUR logró ser percibida desde el exterior como una subregión que adoptaba políticas posneoliberales, proponía más autonomía para sus relaciones internacionales y se alejaba de la influencia estadounidense, intentando inaugurar un ciclo poshegemónico.

Su mayor potencialidad ha sido la voluntad política para contribuir a resolver los conflictos acontecidos a nivel regional. Esta tarea no se realizó a través de mecanismos institucionales preestablecidos, sino fundamentalmente vía los acuerdos presidenciales y las cumbres, o a través de resoluciones adoptadas por los cancilleres del bloque, resaltando el carácter interpresidencial del proceso. Con tales 
mecanismos, la región incrementó su capacidad para solucionar problemáticas propias, desplazando a los espacios interamericanos tradicionales del siglo xx.

Hacia el futuro, el regionalismo/multilateralismo sudamericano enfrenta numerosos desafíos que requerirán de la consolidación de las dinámicas de cooperación tales como la UNASUR. Entre ellos podemos mencionar las nuevas problemáticas de la seguridad, relacionadas con el incremento del narcotráfico y del crimen organizado; la relevancia creciente de la cuestión ambiental; los desafíos a los sistemas democráticos; o bien, los efectos disruptivos de la presencia de China en la región. Como sostiene Kersffeld (2013) se requerirá, por tanto, apelar a la generación de medidas e iniciativas de corte transversal y transterritorial, para dar cuenta de las problemáticas actuales y más acuciantes, así como también de sus posibles soluciones, a fin de ampliar los espacios de inclusión y de generar una UNASUR ciudadana y con un claro y firme horizonte regional.

\section{REFERENCIAS}

Balassa, B. (1961). The Theory of Economic Integration. Westport: Greenwood Press.

Botto, M. (2015). América del Sur y la integración regional: ¿Quo vadis? Los alcances de la cooperación regional en el mercosur. Confines, 21, 9-38.

Bouzas, R., Motta-Vega, P. y Ríos, S. (2007). Crisis y perspectivas de la integración en América del Sur. Foreign Affaris en Español, 61-65.

Busso, A. (2014). Los desafíos de América del Sur frente a Estados Unidos en la segunda década del siglo XXI. XII Semana de Relaçôes Internacionais da
UNESP "Visões do Sul: Crise e Transformaçōes do Sistema internacional”, Brasil.

CEPAL (2011). En busca de una asociación renovada entre América Latina y el Caribe y la Unión Europea. Santiago de Chile: CEPAL. Recuperado de http:// www.cepal.org/publicaciones/xml/0/43260/ En_busca_asociacion_renovada_America_Latina_Caribe_Union_Europea_v2.pdf;

Cepal (2012). La República Popular China y América Latina y el Caribe. Diálogo y cooperación ante los nuevos desafios de la economía global. Santiago de Chile: Cepal. Recuperado de http://www. cepal.org/publicaciones/xml/1/47221/ChinayALCesp.pdf

CEPRID (2009). Injerencia imperial en Bolivia. Recuperado de http://www.nodo50.org/ ceprid/spip. php?article670

Cienfuegos, M. y Sanahuja, J. (coords.) (2010). Una región en construcción. UNASUR y la integración en América del Sur. Barcelona: СІDов.

Comini, N. y Frenkel, A. (2014). Una unasur de baja intensidad. Modelos en pugna y desaceleración del proceso de integración en América del Sur. Nueva Sociedad, 250, 58-77.

Comunidad Sudamericana de Naciones (2004). Declaración de Cusco. III Cumbre Presidencial Sudamericana. Cusco: CsN.

Comunidad Sudamericana de Naciones (2006). Un nuevo modelo de integración de América del Sur, hacia la Unión Sudamericana de Naciones. Bolivia: Comisión Estratégica de Reflexión.

Correa, R. (2013). La Alianza del Pacifico es parte de la estrategia para contrarrestar la integración en América Latina. Recuperado de www.andes. info.ec/es/noticias/presiden-te-correa-alianzapacifco-es-parte-estrategia-contrarrestar-integracion-america 
Diamint, R. (2013). Regionalismo y posicionamiento suramericano: UNASUR y ALBA. Revista $C I D O B$ d'Afers Internacionals, 101, 55-80.

Eje TV (2013). Bolivia, Ecuador y Venezuela son los paises que expulsan ONG. Recuperado de http://eju. tv/2013/12/bolivia-ecuador-y-venezuela-sonlos-pases-que-expulsan-ong/\#sthash.sGvH9m6y. dpuf

Fernández, G. (2013). Espejos y espejismos: las relaciones entre América Latina y Estados Unidos. Nueva Sociedad, 246, 78-86.

Flemes, D. (2012). Regional Leadership in the Global System. Ideas, Interests and Strategies of Regional Powers. Estados Unidos: Ashgate.

Frenkel, A. (2015). Entre conjuras y librepensadores. La UNASUR y la creación de la Escuela Suramericana de Defensa. En XII Congreso Nacional de Ciencia Política. Argentina: Sociedad Argentina de Análisis Político y Universidad Nacional de Cuyo.

Fukuyama, F. (1989). The End of History? The National Interest.

Giaccaglia, C. (2010). La influencia de los actores domésticos en la política exterior brasileña durante el Gobierno de Lula da Silva. Revista Confines, $12,95-121$

Gratius S. y Gomes Saraiva, M. (2013). Continental Regionalism: Brazil's prominent role in the Americas. CEPS Working Document. Recuperado de http://aei.pitt.edu/40231/1/WD_No_374_ Brazil's_Continental_Regionalism.pdf

Grugel, J., Riggirozzi, P. y Thirkell-White, B. E. N. (2008). Beyond the Washington Consensus? Asia and Latin America in search of more autonomous development. International Affairs, 84-3.

Gualdoni, F. y Robossio, A. (2014). América Latina va a dos velocidades. El Pais. Recuperado de http:// economia.elpais.com/economia/2014/06/13/ actualidad/1402670723_367113.html
Haas, E. B. (1971). The Study of Regional Integration: Reflections on the Joy and Anguish of Pretheorizing. En Lindberg L. N. y Scheingold, S. A. (eds.). Regional Integration: Theory and Research (pp. 3-44). Cambridge: Harvard University Press. Hettne, B. y Soderbaum, F. (2002). Theorising the rise of Regionness. En Shaun Breslin, C. H. (ed.). New Regionalism in the Global Political Economy. Theories and cases (pp. 33-47). London: Routledge.

Keohane, R. O. (1990). Multilateralism: An Agenda for Research. International Journal, 45.

Kersffeld, D. (2013). El papel de la unasur ante los conflictos internacionales: dos estudios de caso. Revista Mexicana de Ciencias Politicas y Sociales, 218. Recuperado de http:// www.scielo.org. mx/scielo.php?pid=S018519182013000200010\&script=sci_arttext

Laborde, O. (2014). El rol de las ONG en los planes de EE.UU. Recuperado de http://www.pagina12. com.ar/diario/elmundo/4-252684-2014-08-11. html

Legler, T. (2010). Multilateralism and regional governance in the Americas. En Focal (ed.). Latin America Multilateralism: New directions (pp. 12-17). Canadá: FOCAL. Recuperado de http://www10.iadb. org/intal/intalcdi/PE/2010/06396.pdf

Legler, T. (2013). Post-Hegemonic Regionalism and Sovereignty in Latin America: Optimists, Skeptics, and an Emerging Research Agenda. Contexto Internacional, 2, 181-208.

Legler, T. y Santa-Cruz, A. (2011). El patrón contemporáneo del multilateralismo latinoamericano. Pensamiento Propio, 33, 11-34.

Malamud, A. y Schmitter, P. C. (2006). La experiencia de integración europea y el potencial de integración del Mercosur. Desarrollo Económico. Revista de Ciencias Sociales, 181, 3-31. 
Malamud, A. (2011). Conceptos, teorías y debates sobre la integración regional. Norteamérica. Revista Académica del CISAN-UNAM, 2, 219-249.

Moreira, C., Raus, D. y Gómez Leyton, J. C. (2008). La nueva politica en América Latina. Rupturas y continuidades. Uruguay: Ediciones Trilce.

Oppenheimer, A. (2013). La Alianza del Pacífico saca ventaja al Mercosur. Diario La Nación. Recuperado de http://www.lanacion.com.ar/1586060la-alianza-del-pacifico-saca-ventaja-al-mercosur

Ortiz, G. (2007). Sobre la identidad. Problemas y preguntas. Studia Politice, 10, 35-66.

Oyarzún Serrano, L. (2010). The rol of unasur in Latin American Multilateralism. En FOCAL (ed.). Latin America Multilateralism: New directions (pp. 32-35). Canadá: FocAL. Recuperado de: http:// www10.iadb.org/intal/intalcdi/PE/2010/06396. pdf

Pasquariello Mariano, K. L. y Correa Neto Ribeiro, C. (2015). Regionalismo e Democracia: a participação nos processos de integração na América do Sul. VIII Congreso Latinoamericano de Ciencia Politica, Asociación Latinoamericana de Ciencia Politica (ALACIP). Pontificia Universidad Católica del Perú, Lima.

Rios, S. y Veiga, P. (2007). O'regionalismo pós-liberal, na América do Sul: origens, iniciativas e dilemas. CEPAL Serie Comercio Internacional Integración. Recuperado de http://repositorio.cepal.org/bitstream/handle/11362/4428/S2007612_pt.pdf

Riggirozzi, P. (2010) Region, Regionness and Regionalism in Latin America: Towards a New Synthesis. LATN Working Paper. Recuperado de http:// www19.iadb.org/intal/intalcdi/PE/2012/10382. pdf
Riggirozzi, P. y Tussie, D. (2012). The Rise of PostHegemonic Regionalism. Dordrecht: Springer.

Ruggie, J. (1992). Multilateralism: The Anatomy of an Institution. International Organization, 46-3.

Russell, R. y Tokatlian, J. G. (2008). Resistencia y cooperación: opciones estratégicas de América Latina frente a Estados Unidos. En Lagos, R. (comp.). América Latina: ¿Integración o fragmentación? Buenos Aires: Edhasa.

Sader, E. (2009). El nuevo topo. Los caminos de la izquierda latinoamericana. Buenos Aires: Siglo Veintiuno Editores y CLACSO.

Sanahuja, J. A. (2009). Del 'regionalismo abierto' al 'regionalismo post-liberal'. Crisis y cambio en la integración regional en América Latina. Anuario de la integración regional de América Latina y el Gran Caribe, 7,12-54.

Sanahuja, J. A. (2010). La construcción de una región: Suramérica y el regionalismo posliberal. En Cienfuegos, M., Sanahuja, J. A. (ed.). Una región en construcción. UNASUR y la integración en América del Sur (pp. 87-134). España: Fundación сіров.

Sanahuja, J. A. (2012). Regionalismo post-liberal y multilateralismo en Sudamérica: el caso de UNASUR. En Serbin, A., Martínez, L. y Ramanzini, H. J. (coords.). El regionalismo "post-liberal" en América Latina y el Caribe: Nuevos actores, nuevos temas, nuevos desafios (pp. 19-72). Argentina: CRIES.

Serbin, A. (2010). De despertares y anarquías. En Foreign Affairs Latinoamérica, 3, 6-11.

Zakaria, F. (2008) The post-american world. New York: Norton Books. 